\title{
Hypoglycaemia and glucose sensing
}

\author{
D. B aker ${ }^{1}$, M . E vans ${ }^{2}$, P. Cryer ${ }^{2}$, R . Sherwin ${ }^{4}$ \\ ${ }^{1}$ Department of Bioengineering, University of California, San Diego, California, USA \\ ${ }^{2}$ Department of Medicine, Kings College School of Medicine and Dentistry, London, UK \\ ${ }^{3}$ Washington University School of Medicine, St. Louis, Missouri, USA \\ ${ }^{4}$ Department of Internal Medicine, Yale University School of Medicine, New Haven, Connecticut, USA
}

Hypoglycaemia is an important consequence of diabetes mellitus. Risk factors for severe hypoglycaemia include intensified insulin therapy (with a threefold increase observed in the Diabetes Control and Complications Trial [DCCT]) and hypoglycaemia unawareness, caused by hypoglycaemia itself. This article reviews: 1) the sensing process for severe hypoglycaemia (which is probably carried out by the ventromedial hypothalamus); 2) the reversible impairment of hypoglycaemia awareness and the counterregulatory responses; 3 ) the role of cerebral blood flow and metabolism; and 4) progress on the development of continuous glucose monitoring devices to warn of hypoglycaemia.

\section{Stephanie Amiel: Prevalence and prevention of hypoglycaemia}

The frequency of hypoglycaemia is difficult to assess because of variations in the methods of describing hypoglycaemia and under-reporting, even for severe episodes. The definition of severe hypoglycaemia in the literature varies from episodes requiring third party rescue to episodes of frank coma only. Reported rates for adults vary from 1.6 severe hypoglycaemic episodes per patient year down to 0.2 events per patient year in the conventional treatment

\footnotetext{
Participants: S. Amiel, Department of Medicine, King's College School of Medicine and Dentistry, London, UK

D. Gough, Department of Bioengineering, University of California, Dan Diego, California, USA

A. Nehlig, INSERM U398, Strasbourg, France

G. Reach, Diabetes Department, Hotel-Dieu, Paris, France
}

Corresponding author: D. A. Baker, Ph.D., Department of Bioengineering, Institute for Biomedical Engineering, University of California, San Diego, 9500 Gilman Drive, La Jolla, California 92093-0412, USA arm of the DCCT. Risk factors for severe hypoglycaemia are increasing duration of diabetes, increasing age, intensified insulin therapy (a threefold increase in the prospective DCCT and Stockholm studies), a history of severe hypoglycaemia, loss of symptomatic awareness of hypoglycaemia, sleep, alcohol and "tight" glycaemic control. In children the reported rates of severe hypoglycaemia are less than in adults with rates of between 0.2 and 0.4 events per patient year quoted, although the reported frequency may be rising. However, this may reflect differences in symptoms experienced by children and their ability to report them leading to under-reporting of hypoglycaemia in children. Additionally, higher ambient glucose levels and the maintenance of some degree of C-peptide secretion may contribute to lower rates in children.

D efective glucose counterregulation. Glucagon secretory responses to hypoglycaemia are lost in patients with established (C-peptide negative) insulin-dependent diabetes mellitus. The mechanism of this key defect is unknown. In the setting of absent glucagon responses, reduced adrenaline responses cause defective glucose counterregulation, a syndrome shown prospectively to be associated with substantially increased rates of severe hypoglycaemia. Recent antecedent hypoglycaemia plays an important role in the pathogenesis of the reduced adrenaline responses, but the mechanism is unknown. Increased blood-tobrain glucose transport has been postulated.

$\mathrm{H}$ ypoglycaemia unawareness. For patients with diabetes, the main defense against severe hypoglycaemia is subjective recognition of the onset of the glucose fall. Some patients lose this symptomatic awareness, and in conjunction show reduced counterregulatory responses (specifically adrenomedullary adrenaline responses) to hypoglycaemia. The principal defect is 
the lowering of blood glucose levels at which symptoms and counterregulatory hormone responses are initiated. These patients have been shown prospectively to be at an increased risk of severe hypoglycaemia. There is now considerable evidence that it is antecedent hypoglycaemia per se which causes these adaptations in glucose thresholds, with a lower blood glucose being required to trigger counterregulatory hormone responses and symptoms. Although there may be some hepatic glucose sensing, the main physiological sensing of hypoglycaemia in the body seems to be located within the brain. This implies that the brain is able to "adapt" to preceding hypoglycaemia and behave more normally during subsequent hypoglycaemia. The clinical syndrome of hypoglycaemia unawareness suggests, however, that cognitive areas of the brain are not able to adapt in the same way as glucose sensing areas, resulting in cognitive impairment occurring before or with counterregulatory and symptomatic responses; alternatively, symptoms may simply be lost. Either way, by the time symptoms occur the subject is too confused to take appropriate action. Studies of cognitive impairment during hypoglycaemia have shown conflicting results, some showing that thresholds for cognitive dysfunction are "preserved" (i.e. fixed at the same glucose threshold regardless of preceding glycaemic experience) whereas others have found some adaptation to preceding hypoglycaemia. Some of these disparities, however, may be a result of using different methods of assessing cognitive function during hypoglycaemia. Different cognitive tests will assess different brain regions, which in turn may vary in both their susceptibility to hypoglycaemia, and potentially in their ability to adapt to previous glycaemic experience.

The adaptation of glucose thresholds may be reversible. Counterregulatory and symptomatic responses to hypoglycaemia in subgroups of patients with hypoglycaemia unawareness may be at least partly restored by scrupulous avoidance of hypoglycaemia. Clinical studies have achieved this by intensive clinical follow-up, paying particular attention to such risk factors as exercise, alcohol, missed meals and inappropriate insulin dosage/timing. Nocturnal hypoglycaemia is common and is often unrecognized, but may be an important contributor to hypoglycaemia unawareness. More recent strategies for protecting against nocturnal hypoglycaemia include moving the nocturnal intermediate acting insulin to bedtime, the use of short acting insulin analogues such as Lispro for the evening meal, the elevation of pre-bedtime blood glucose levels to greater than $7 \mathrm{mmol} / 1$ and possible changes in the composition of the bedtime snack to allow slower glucose absorption.

The mechanisms by which glucose thresholds adapt remain obscure. It has been widely suggested that the adaptation may occur by alteration of blood brain glucose transporter density. Some studies in rodents have reported increased GLUT1 glucose transporters (responsible for blood-brain barrier transport) after preceding chronic hypoglycaemia and starvation, and downregulation in streptozotocin-induced diabetes, although this is not a universal finding. Unpublished data from in vivo studies using the technique of positron emission tomography (PET) scanning using the positron emitting isotope $1-{ }^{11} \mathrm{C}$ labelled glucose in six subjects with poorly controlled IDDM (mean $\mathrm{HbA}_{1 \mathrm{c}} 10.1 \%$ ) were presented. No differences were found between patients and control subjects during mild hypoglycaemia $(3.6 \mathrm{mmol} / \mathrm{l})$ in blood to brain glucose transport, cerebral metabolic rate of glucose or cerebral blood flow. There was, however, a reduced adrenaline response to hypoglycaemia in the diabetic group, a finding not explicable on the basis of increased blood to brain glucose transport since the latter was not detected.

Astrid Nehlig, with comments by Phillip Cryer: Cerebral glucose utilization and blood flow during hypoglycaemia

The physiological changes seen during hypoglycaemia in cerebral glucose metabolism and blood flow are unique when compared with other situations such as the changes seen during development in early life. As humans and young rats develop, cerebral glucose utilization and blood flow (measured by PET scanning in human studies and by autoradiography in animal work) rise to peak during maximum brain development (between 4 and 9 years of age in humans). Changes in glucose utilization and blood flow tend to mirror one another.

During hypoglycaemia this is not the case. Cerebral blood flow increases during severe hypoglycaemia, but cerebral metabolism of glucose falls. This fall in glucose metabolic rate shows regional differences between different brain areas which have been examined in animals by the technique of ${ }^{14} \mathrm{C}-2$ deoxyglucose autoradiography. The neocortex, hippocampus and caudate/putamen were found to be particularly sensitive, and the thalamus, hypothalamus, brain stem and cerebellum were more resistant to hypoglycaemia. Similarly cerebral blood flow increased differentially in different brain regions during the onset of hypoglycaemia. PET scanning offers the exciting potential opportunity to examine these regional differences in human studies.

A Iternate non-glucose substrates. Autoradiographic studies in developing rats also demonstrate the ability of mammalian brain to use non-glucose substrates. Suckling rats are able to use significant quantities of beta-hydroxybutyrate in addition to glucose. The uptake of beta-hydroxybutyrate is relatively homogeneous in comparison to the heterogeneity of glucose 
uptake measured by autoradiography. After weaning the uptake of beta-hydroxybutyrate falls. Studies in humans have shown that beta-hydroxybutyrate (and lactate) can at least partly substitute for glucose during acute experimental hypoglycaemia.

Microdialysis studies in human subjects undergoing intracerebral depth electrode monitoring for intractable epilepsy show that brain glucose levels are consistently $30 \%$ of circulating plasma values. In contrast, brain extracellular fluid (ECF) lactate levels are 4 to 5 times higher than blood values. It is possible that these high brain ECF values may protect neurons against hypoglycaemia by acting as a temporary fuel source. It has been suggested in the neuroscience literature that glial cells may be involved in this glucose/lactate cycling, although this remains to be established.

\section{Robert Sherwin: The site of glucose sensing}

There is now considerable evidence that the predominant glucose sensing mechanism is located in the ventromedial hypothalamus (VMH), although evidence for extracerebral sensors, particularly in the liver, has been reported. Studies using microdialysis probes to alter the local intracerebral environment have been used to investigate the $\mathrm{VMH}$. Lesioning the VMH with ibotenic acid reduced counterregulatory responses in rats. Similarly, instilling glucose locally to the VMH attenuated the responses to systemic hypoglycaemia, whereas the glucose analogue 2deoxyglucose, which induces local glucopenia in the $\mathrm{VMH}$, stimulated a brisk counterregulatory response. When the alternate substrate L-lactate was perfused locally, counterregulatory responses were blunted compared with D-lactate. Taken together these studies suggest that the $\mathrm{VMH}$ is involved not just in the efferent pathway of counterregulatory responses but is also actively involved in sensing hypoglycaemia. The response to L-lactate suggests that the sensor acts as a "fuel" sensor rather than as a pure glucose sensor.

If the $\mathrm{VMH}$ is the predominant glucose sensor, one might expect to see changes in the VMH responses to hypoglycaemia depending on previous glycaemic experience. Preliminary data were reported that in rats made recurrently hypoglycaemic, both glucagon and adrenaline responses to 2-deoxyglucose VMH perfusion were nearly totally suppressed, suggesting an inability of the VMH to recognise hypoglycaemia and/or activate counterregulation. Thus impaired counterregulation during intensive insulin therapy may result from alterations of the $\mathrm{VMH}$ or its distal pathways.

The mechanism of glucose sensing. An intriguing hypothesis suggested by Dr. Sherwin is that the VMH glucose sensor may be detecting glucose flux in the same way as the pancreatic beta cell. There is some experimental evidence for this. Cells in the medial hypothalamus express glucokinase and perhaps GLUT2, and they contain ATP-sensitive potassium channels $\left(\mathrm{K}_{\mathrm{ATP}}\right)$. Moreover microdialysis probes were used to examine whether the rat substantia nigra, an area rich in $\mathrm{K}_{\mathrm{ATP}}$ channels, could respond to changes in blood glucose. Perfusing with glucose or the sulphonylurea glipizide increased g-aminobutyric acid (GABA) release locally. Lemakalim (a $\mathrm{K}_{\mathrm{ATP}}$ channel activator) and 2-deoxyglucose had the opposite effect, as did systemic hypoglycaemia. Thus, there may be cells in the substantia nigra that behave much like beta cells. However, their effecter molecule is GABA rather than insulin.

\section{Continuous glucose monitoring devices}

The above considerations suggest that there is a need for a glucose sensing device that can function as a hypoglycaemia alarm. To be effective for hypoglycaemia detection, this sensing system should operate continuously and not rely on the initiative of the user for sample collection. A continuous glucose sensor would also be of great value in the management of hyperglycaemia and, in conjunction with a controllable insulin delivery system, may lead to much closer blood glucose control. It is desirable to exploit the continuous glucose sensor and the mechanical artificial pancreas as near-term treatment strategies to provide a bridge to a time when other forms of therapy discussed elsewhere in this publication may become available. Many issues related to the development of new glucose sensors have been recently reviewed [1].

\section{Gérard Reach: Enzyme electrode sensors based on hydrogen peroxide detection}

One type of glucose sensor is an acute subcutaneous implantable sensor based on the enzyme electrode principle and detection of hydrogen peroxide, such as that being developed by Dr. Gérard Reach and his colleagues [2], and several other groups. This system consists of a small, needle-like sensor to be placed under the skin by the patient himself on a 37 day basis, and of an electronic control unit (ECU) for processing the sensor output.

This sensor has been fabricated at the size of an insulin injection needle, is flexible, and can be implanted through a 21 gauge needle in the subcutaneous tissue. The enzyme glucose oxidase is layered near, but not on the tip of the sensor. Thus, there is less risk for damage of the outer layer during implantation or explantation of the sensor. The cathode is a 
silver-silver chloride wire wrapped around the sensor. An internal layer is a composite Nafion-Cellulose Acetate membrane and the outer layer is composed of polyurethane.

The team has developed a wearable, battery-driven ECU, which controls the sensor potential, stores and processes the current. An in vivo calibration procedure is made possible by entering into the device the values of two different blood glucose concentrations and the corresponding currents, during a temporary connection of the ECU to a portable personal computer. Signal processing is performed by sophisticated algorithms based on mathematical morphology techniques.

Data obtained by the group indicate that the sensor has an in vitro sensitivity around $2-4 \mathrm{nA} \cdot \mathrm{mmol} / \mathrm{l}$, and is largely insensitive to ascorbate, urate, and acetaminophen. It has an in vitro stability of several weeks under continuous functioning. Sensors can be stored in a dry state for several weeks after sterilization. Data have been generated by implanting sensors in the subcutaneous tissue of rats, dogs, and human volunteers.

A two-point in vivo calibration procedure has been developed for transforming the sensor output into an estimation of glucose concentration. By this method, the blood glucose level is changed by administering glucose (or during a meal) and two blood glucose levels are measured. By comparing them with corresponding values of the current, it is possible by linear extrapolation to determine an in vivo sensitivity, S, and an extrapolated background current, Io, that would be observed in vivo in the absence of glucose, and then to apply these parameters to the current, I, to get an estimation of the subcutaneous glucose concentration as $\mathrm{G}=(\mathrm{I}-\mathrm{Io}) / \mathrm{S}$. This procedure has been validated with a glucose sensor implanted in the subcutaneous tissue of rats, dogs, and human volunteers. Changes in the estimation of glucose concentration in the subcutaneous tissue was shown to follow those in blood with a lag time usually shorter than 5$10 \mathrm{~min}$. The changes in the subcutaneous glucose concentration follow those in blood with a very short delay, in general shorter than $5 \mathrm{~min}$. In vivo, the domain of linearity of the sensor response to glucose, i. e. the concentration range in which the sensor's sensitivity is constant, was found to be at least $15 \mathrm{mmol} / \mathrm{l}$. It was possible to demonstrate that the sensor can be calibrated when blood glucose concentration is measured with a glucometer in whole blood and not solely with a Beckman Analyzer on plasma.

Dr. Reach's group recently made the observation that during a decrease in blood glucose concentration, induced either by exogenous insulin administration or by endogenous insulin (for instance during the decrease in blood glucose concentration of the second phase of an oral glucose tolerance test), the decrease in the current generated by the sensor is observed before the decrease in blood glucose concentration [3]. This was observed when the glucose sensor was implanted in the subcutaneous tissue of rats, dogs, and human volunteers. This effect may be due to the fact that insulin pulls glucose from the interstitial space, where the sensor is implanted, into surrounding cells, and thus represent the direct observation of the peripheral effect of insulin.

Finally, the research group recently developed software for sensor calibration based on recognizing a sensor output plateau, which triggers a request for blood glucose determination. The ECU is now able to compare its estimation of glucose concentration to a hypoglycaemic threshold, which can be set by the user, and to trigger an alarm. The plateau recognition software and the hypoglycaemic alarm system have been validated in experiments performed in rats. It is now necessary to address the following using clinical trials performed in diabetic volunteers: 1) longevity of the sensor; 2) need for re-calibration; 3 ) the relationship between blood and subcutaneous glucose concentration, especially during or after hypoglycaemia, and during exercise. The next step will be the development of methods for sensor mass production, the full miniaturization of the ECU, and the development of telemetry connection between the sensor and the ECU. This will make possible the implementation of full scale clinical trials aimed to carefully evaluate the system.

\section{David Gough: Implantable enzyme electrode sensors based on oxygen detection}

Glucose sensors based on glucose oxidase coupled to oxygen sensors are being developed by Dr. David Gough and his colleagues. The sensors incorporate an oxygen reference sensor and temperature sensor and have been made in several configurations for either implantation in subcutaneous tissues or chronic intravenous implantation. Instrumentation for sensor operation has been developed in the form of both a portable external unit and a totally implantable telemetry unit. The development has been based on systematic engineering and in vivo experiments in animals.

As a short-term subcutaneous implant, the sensor produces signals that are difficult to interpret in terms of blood glucose concentration. This is a result of the interaction between the implanted sensor and local subcutaneous tissue, about which there is surprisingly little information. The sensor response to blood glucose is affected by lags, noise, and artifacts due to variable local perfusion, etc. This response has been characteristic of this and other subcutaneous sensors, and confounds the determination of blood glucose concentration. The goal of this research group has been to develop a mechanistic understanding of the 
relationship between blood glucose concentration and signal, rather than a statistical correlation. Such an understanding may suggest modes of sensor operation that do not require recalibration. Studies focus on the role of local physiologic factors, such as tissue architecture, microvascular perfusion, tissue oxygen distribution, movement, implant biocompatibility, and wound healing. This work involves novel optical instrumentation for visualization of the microvasculature and scanning of substrate levels of living tissues. Preliminary research has suggested promising approaches to account for the effects of the tissue environment, but further research is necessary.

In contrast, a flexible, catheter-like configuration of the sensor for chronic intravenous implantation has produced a much more straightforward response to blood glucose concentration in animal studies. This version of the sensor has operated continuously over 100 days in dogs with implanted telemetry, with no adverse effects or need for recalibration [4]. The lowest glucose level used in this study was approximately $2.7 \mathrm{mmol} / \mathrm{l}$, the euglycaemic level in these dogs, to which the sensor readily responded. In a possible clinical configuration, the sensor would be nonsurgically introduced into the superior vena cava much the way pacemaker leads and chronic hyperalimentation catheters are presently placed. The sensor and catheter would connect to a permanently implanted telemetry/contact module that contains a long-term battery, electronic instrumentation and telemetry unit for transmission of signals to an external, belt-mounted receiver or beeper. Sensor replacement would be carried out by a non-surgical procedure every 3 to 6 months, prior to the need for sensor recalibration. The expectations for safety in implementation of this intravenous strategy should be comparable to the low incidence of untoward events documented in chronic hyperalimentation catheter use. Although no basic research is required for implementation of this sensor configuration in humans, there is a need for a coordinated developmental programme to combine and implement existing technologies. This effort may involve engineering development and design facilities, private or governmental fabrication and testing facilities, instrumentation designers, and clinical testing facilities. Developmental questions include the issue of safety of implantation in diabetic patients, manufacture of sensors and instrumentation, and further evaluation. With adequate resources, implementation of this technology in the near future is a realistic goal.

O ther glucose sensor principles. Alternative glucose sensing approaches should be pursued but are farther in the future. A cute microdialysis systems are presently being used in some patients, but require management of considerable external paraphernalia and may not be suitable for many patients. There is a need to understand better glucose transport to the probe at a basic level. Transdermal extraction of tissue fluids for glucose assay based on iontophoresis, sonophoresis, applied vacuum, solvents or other strategies is at a very preliminary stage and requires more basic research into the fluid extraction process, glucose assay methods for very low concentrations in very small volumes, and system safety. Non-invasive technologies using near-infrared spectroscopy, optical dispersion effects, and certain other optical principles seem unlikely to be developed in the near future. There are substantial difficulties in demonstrating specificity to glucose, accounting for the effects of tissues, and calibration. Optical sensors require more basic research before demonstration of feasibility and subsequent development.

\section{Conclusions}

There have been rapid advances in our knowledge about the mechanism by which the body senses hypoglycaemia. The VMH is an important site of glucose sensing. The mechanisms by which this occurs remain to be determined but an intriguing possibility is that this sensing is analogous to glucose sensing by the pancreatic beta cell. Similarly we are also beginning to understand how the sensing mechanism may be altered by preceding hypoglycaemia, hyperglycaemia or diabetes per se. This knowledge is essential if we are to devise therapeutic strategies to protect against hypoglycaemia.

Contemporary clinical management should be directed towards avoiding hypoglycaemia with the same rigour as currently directed towards avoiding hyperglycaemia, particularly in patients with hypoglycaemia unawareness or other identifiable risk factors for severe hypoglycaemia. Intensified insulin therapy is a useful tool for diminishing the risk of the chronic complications of hyperglycaemia, but it clearly causes an increased risk of hypoglycaemia and glycaemic targets should be individualised accordingly.

There are significant possibilities for the implementation of continuous implantable glucose sensors in the near future. Sustained research and development of several sensor approaches should be cultivated, focusing on realistic, near-term sensor strategies. The first application may be the development of a hypoglycaemic alarm. These systems may also lead to the development of an artificial beta cell.

\section{Future directions and recommendations}

1. Therapeutic strategies that will minimize the risk of iatrogenic hypoglycaemia and thus permit lower mean glycaemia for prevention or delay of complications of diabetes are of immediate need for people 
with diabetes. Since iatrogenic hypoglycaemia is the result of the interplay between absolute or relative therapeutic insulin excess and compromised endogenous glucose counterregulatory systems, these strategies could include more physiological insulin replacement, measures that prevent, correct or compensate for compromised hormonal and behavioural defences against developing hypoglycaemia, or both. While some approaches can be envisioned on the basis of current knowledge, substantial therapeutic advances will require greater understanding of the fundamental mechanisms of glucoregulatory physiology, and particularly its pathophysiology in people and experimental animals with diabetes.

2. Methods to replace insulin such as a closed loop insulin delivery system, islet cell transplantation, implantable cells that provide glucose-regulated insulin secretion, or other approaches are needed for restoration of euglycaemia without substantial risk of hypoglycaemia. This would prevent the specific longterm complications of diabetes (retinopathy, nephropathy and neuropathy) and, when coupled with comprehensive medical care, would likely reduce atherosclerotic risks.

3. Due to the therapeutic need, it is important to focus on implementation of near-term treatment strategies, rather than speculate on the potential advantages of distant approaches. In any case, development of a variety of treatment strategies must be pursued. Some examples of areas needed to be studied in the short term are:

- Elucidation of the mechanisms for the absent response of glucagon to hypoglycaemia in IDDM

- Understanding of how and why IDDM per se impairs activation of autonomic responses, including adrenaline responses, to hypoglycaemia, and how they may be restored

- Identification of the cellular and molecular mechanisms for the suppressive effect of iatrogenic hypoglycaemia on counterregulatory responses so that reversal strategies can be developed

- Study of the frequency and pathogenesis of nocturnal hypoglycaemia so that better methods of prevention can be developed

- Establishment of better methods for measuring brain metabolic dysfunction, particularly regional changes

- Gain a better understanding of the long-term effects of hypoglycaemia on brain function and how they are affected by age and IDDM duration

- Development of a hypoglycaemic alarm based on near-term sensor technology

- Development of methods for assessing subcutaneous glucose dynamics so as to allow interpretation of glucose sensor signals and better calibration of sensing devices

- Development of a closed loop system with an accurate glucose sensing device, controller and an insulin delivery system

- Basic research on more distant approaches for glucose detection such as tissue fluid extraction, optical methods, etc.

\section{References}

1. Gough DA, Armour JC (1995) Development of the implantable glucose sensor: what are the prospects and why is it taking so long? Diabetes 44: 1005-1009

2. Poitout V, Moatti-Sirat D, Reach G et al. (1993) A glucose monitoring system for on line estimation in man of blood glucose concentration using a miniaturized glucose sensor implanted in the subcutaneous tissue, and a wearable control unit. Diabetologia 36: 658-665

3. Thomé-Dure V, Reach G, Gangnerau MN et al. (1996) Use of a subcutaneous glucose sensor to detect decrease in glucose concentration prior to observation in blood. Analytical Chemistry 68: 3822-3826

4. Armour JC, Lucisano JY, McKean BD, Gough DA (1990) Application of a chronic intravascular blood glucose sensor in dogs. Diabetes 39: 1519-1926 